\title{
Prevalence and risk of chronic kidney disease in oral lichen planus: a large cross-sectional study from eastern China
}

\author{
Yiwen Deng ${ }^{1 \#}$, Chengyi Wang ${ }^{1 *}$, Yeqing Shen ${ }^{1 *}$, Bo Shen $^{2}$, Feng Ding ${ }^{2}$, Guoyao Tang ${ }^{1}$, Wei Liu ${ }^{3}$ \\ ${ }^{1}$ Department of Oral Mucosal Diseases, Shanghai Ninth People's Hospital, Shanghai Jiao Tong University School of Medicine, College of \\ Stomatology, Shanghai Jiao Tong University, National Center for Stomatology, National Clinical Research Center for Oral Diseases, Shanghai Key \\ Laboratory of Stomatology, Shanghai, China; ${ }^{2}$ Department of Nephrology, Shanghai Ninth People's Hospital, Shanghai Jiao Tong University School \\ of Medicine, Shanghai, China; ${ }^{3}$ Department of Oral and Maxillofacial-Head and Neck Oncology, Shanghai Ninth People's Hospital, Shanghai Jiao \\ Tong University School of Medicine, Shanghai, China \\ Contributions: (I) Conception and design: G Tang, W Liu; (II) Administrative support: Y Deng, G Tang; (III) Provision of study materials or patients: \\ Y Deng, B Shen, F Ding; (IV) Collection and assembly of data: C Wang, Y Shen; (V) Data analysis and interpretation: Y Deng, W Liu; (VI) \\ Manuscript writing: All authors; (VII) Final approval of manuscript: All authors. \\ \#These authors contributed equally to this work. \\ Correspondence to: Dr. Guoyao Tang. Department of Oral Mucosal Diseases, Shanghai Ninth People's Hospital, 500 Quxi Road, Shanghai 200011, \\ China. Email: g.tang@163.com; Dr. Wei Liu. Department of Oral and Maxillofacial-Head and Neck Oncology, Shanghai Ninth People’s Hospital, \\ 639 Zhizaoju Road, Shanghai 200011, China. Email: liuweb@hotmail.com.
}

Background: Oral lichen planus (OLP) is a chronic inflammatory mucocutaneous disease which is frequently associated with comorbidities such as diabetes and cardiovascular diseases. However, little is known about the association of OLP with impaired kidney function. To elucidate the possible association of chronic kidney disease (CKD) with OLP and its severity, this study investigated the prevalence of CKD as well as its risk factors in patients with OLP.

Methods: A large prospective cross-sectional study of 1,021 patients with OLP was carried out using questionnaires and laboratory tests available from an oral medicine clinic at a university in eastern China. According to the Kidney Disease: Improving Global Outcomes (KDIGO) diagnostic guideline, CKD was classified based on the estimated glomerular filtration rate (eGFR, $<60 \mathrm{~mL} / \mathrm{min} / 1.73 \mathrm{~m}^{2}$ ) or urinary albumin to creatinine ratio (UACR, $>30 \mathrm{mg} / \mathrm{g}$ ).

Results: The prevalence of CKD in the patients with OLP in this study was $14.3 \%$ (95\% CI, 12.3-16.6\%), which was higher than that in the general Chinese population (10.8\%; 95\% CI, 10.2-11.3\%). The mean values of serum creatinine, eGFR, UACR, and urine $\mathrm{N}$-acetyl- $\beta$-D-glucosidase in patients with CKD were significantly higher than those in patients without CKD (all $\mathrm{P}<0.01$ ). Pearson's correlation analysis revealed that CKD stage and UACR were positively correlated with the severity of OLP (both $\mathrm{P}<0.001$ ). Importantly, multivariate regression analysis revealed that age $\geq 58$ years old, female sex, and hypertension were independent risk factors for incident CKD and abnormal UACR ( $>30 \mathrm{mg} / \mathrm{g}$ ) in patients with OLP (all $\mathrm{P}<0.01)$.

Conclusions: This study has reported for the first time that CKD is a comorbidity in patients with OLP. The occurrence and staging of incident CKD are associated with OLP and its severity.

Keywords: Chronic kidney disease (CKD); oral lichen planus (OLP); hypertension; albuminuria; estimated glomerular filtration rate (eGFR)

Submitted Feb 10, 2021. Accepted for publication Apr 16, 2021.

doi: 10.21037/atm-21-699

View this article at: http://dx.doi.org/10.21037/atm-21-699 


\section{Introduction}

Lichen planus (LP) is a common chronic inflammatory mucocutaneous disease of probable autoimmune-related etiology $(1,2)$. Oral LP (OLP) affects $0.38 \%$ to $2.0 \%$ of the general population, occurring mostly in female, middle-aged, and older adults (3). OLP is characterized by a T-cell-mediated immune response against epithelial cells, causing epithelial cell damage and subepithelial persistent accumulation of $T$ lymphocytes (1). In its clinical presentation, OLP ranges from asymptomatic reticular white lichen to symptomatic atrophic-erosive red lichen with symptoms of burning, irritation, and pain (4). Despite a variety of treatments being available, this disease often severely affects patients' quality of life due to its protracted clinical course $(4,5)$ and, in particular, its potential for malignant transformation $(6,7)$. Although the etiology and pathogenesis of OLP remain unclear, it has been reported to be associated with several systemic comorbidities including diabetes mellitus (8) and cardiovascular disease (CVD) (9). Furthermore, it is noteworthy that in clinical practice, a proportion of patients with OLP suffer from renal dysfunction, such as abnormal serum creatinine and urine albumin levels (Figures S1,S2).

Chronic kidney disease (CKD), which includes a variety of renal dysfunction diseases, is a major health problem worldwide due to its high rates of mortality and comorbidity (10). Based on classification by the Kidney Disease Improving Global Outcome (KDIGO) guideline, the prevalence of CKD in China was reported to be $10.8 \%$ by one well-recognized study (11). Recently, CKD has been reported to be associated with some chronic inflammatory disorders, such as periodontitis $(12,13)$, rheumatoid arthritis (14), and psoriasis (15). Emerging evidence suggests that immune systems and its components have important roles in the initiation, progression and complications of CKD by systemic inflammation (16). CKD patients are characterized by a complex impairment of the immune system, which combines low-grade chronic inflammation and the inability to mount protective immune responses (17). This chronic inflammation arises possibly from accumulation of proinflammatory cytokines in CKD patients, whom have been characterized by variable accumulation and aberrant activation status of memory $\mathrm{T}$ cell subsets $(18,19)$. It is reasonable to assume that aberrant immune response, with an emphasis on $\mathrm{T}$ cell-mediated immune dysfunction, related to the potential mechanisms that might link both OLP and CKD. Furthermore, studies on LP have revealed a possible association with hyperuricemia and abnormal creatinine $(20,21)$, with the authors also stating that the involvement of metabolic defects was highly detected in patients with LP compared to controls $(20,21)$. These observations, along with our clinical experience treating patients with OLP, suggest a possible association between kidney dysfunction and LP.

At present, little is known about the association between LP and renal function impairment. Therefore, to examine this relationship, we conducted a large prospective crosssectional study using the questionnaires and laboratory tests available from an oral medicine clinic at a university in eastern China. We aimed to investigate the prevalence of CKD and its risk factors in patients with OLP in order to elucidate the possible association of CKD with OLP and its severity. We present the following article in accordance with the STROBE reporting checklist (available at http:// dx.doi.org/10.21037/atm-21-699).

\section{Methods}

\section{Participants}

At the time this study was planned, no previous study had evaluated the association between CKD as the primary exposure and OLP as the outcome. We estimated the sample size based on the sample size $(n=47,204)$ in a nationally representative cross-sectional survey of the Chinese population (11). Based on the highest estimated prevalence $(2.0 \%)$ of OLP in the general population (3), we assumed that 944 individuals $(2.0 \%$ of 47,204$)$ had OLP. Taking into consideration an alpha of $5 \%$ and a relative error of sampling of $10 \%$, we calculated that the present study would require a sample size of 1049 . This study was approved by the Institutional Review Board of Shanghai Ninth People's Hospital, Shanghai Jiao Tong University School of Medicine [No. (2016)01]. Written informed consent was obtained from all study participants. The study was conducted in accordance with the Declaration of Helsinki (as revised in 2013). Patients with a diagnosis of OLP who initially visited the clinic at the Department of Oral Mucosal Diseases of our hospital between April 2016 and March 2018 were prospectively enrolled into this prospective cross-sectional study.

The inclusion criteria for patients were as follows: (I) aged $\geq 18$ years; (II) a clinical and histological diagnosis of OLP which met the modified World Health Organization (WHO) diagnostic criteria (22); and (III) agreed to participate 
in the study. The exclusion criteria were as follows: (I) patients who were pregnant; (II) patients diagnosed with periodontitis with a periodontal probing depth of $\geq 6 \mathrm{~mm}$ and clinical attachment loss of $\geq 6 \mathrm{~mm}$; (III) patients with a history of malignancy or other inflammatory or autoimmune diseases such as psoriasis, vitiligo, Behçet's disease, lupus erythematosus, or rheumatoid arthritis; and (IV) patients who had taken antibiotics, or immunosuppressive or nephrotoxic drugs in the 6 months prior. The antibiotics include aminoglycosides, cephalosporins, quinolones, antitubercular agents; the immunosuppressive drugs include steroids, cyclophosphamide, azathioprine, cyclosporine, methotrexate, chloroquine; the nephrotoxic drugs include non-steroidal anti-inflammatory drugs, lithium, chloroquine, cytotoxic drugs e.g., cisplatinum.

\section{Data collection}

The age, sex, and body mass index (BMI) of each participant were recorded. Any medical history of kidney diseases, hypertension, diabetes, or CVD was also recorded, on a standardized computerized database, before laboratory tests of renal function. Blood and urine samples $(10 \mathrm{~mL})$ were collected from all participants, and all analyses were carried out at the Department of Clinical Laboratory of our hospital. Serum creatinine was examined using Siemens Dimension RxL Max HM, serum cystatin $C$ and urine $\mathrm{N}$-acetyl- $\beta$-D-glucosidase (UNAG) were examined using Siemens ADVIA 2400, urinary albumin, urinary creatinine, and C-reactive protein were examined using Dade Behring BN ProSpec according to these manufacturers' protocols. All laboratory tests were completed on the basis of a standardization and certification programme.

According to the KDIGO stratification risk criteria (23) and the calculation method previously described (11), CKD was classified based on the estimated glomerular filtration rate (eGFR $<60 \mathrm{~mL} / \mathrm{min} / 1.73 \mathrm{~m}^{2}$ ) or urinary albumin to creatinine ratio (UACR $>30 \mathrm{mg} / \mathrm{g}$ classified as albuminuria). The stratified staging of CKD was also assessed based on the eGFR and UACR according to the classification of the KDIGO statement (23). Further, the disease severity of OLP was assessed using the Thongprasom sign scoring system (24), which has been used in previous studies (25-27). Using this scoring system, patients are given a score of: 0 for normal healthy mucosa; 1 for lesions with only white striae; 2 for mixed keratotic and atrophic or erythematous lesions $<1 \mathrm{~cm}^{2}$ in size; 3 for keratotic and atrophic or erythematous lesions $>1 \mathrm{~cm}^{2}$ in size; 4 for erosive/ulcerative lesions $<1 \mathrm{~cm}^{2}$; and 5 for erosive/ ulcerative lesions $>1 \mathrm{~cm}^{2}$.

\section{Statistical analysis}

When the expected values were less than 5 , differences in quantitative and qualitative variables between 2 groups were calculated by the Student's $t$ test and the chi-square $\left(\chi^{2}\right)$ test or Fisher's exact test, respectively. Differences in quantitative and qualitative variables between multiple groups were calculated using 1-way analysis of variance and non-parametric tests, respectively. If quantitative variables did not follow a Gaussian distribution, the differences between 2 groups were calculated using the non-parametric test. Pearson's correlation analysis was used to determine the correlation between CKD stratified risk and OLP severity. Logistic regression with random effects model analysis was applied to evaluate odds ratios (OR) with $95 \%$ confidence intervals (CIs) and the associations among variables. In the logistic regression, univariate analysis was first performed to obtain the significant variables. To further assess and adjust the influence of each significant variable, multivariate analysis was subsequently performed, and the factors that remained statistically significant were determined. Statistical analysis was performed using SPSS for Windows (version 23.0; SPSS Inc.). All tests were 2-sided, and $P$ values of $<0.05$ were considered to be statistically significant.

\section{Results}

\section{Prevalence of CKD in OLP and renal function analysis}

A total of 1,021 eligible patients with OLP, who completed the blood and/or urine laboratory tests, were consecutively enrolled in this prospective cross-sectional study. Among the 1,021 patients were 352 males and 669 females, and the average age was 50.4 years (range, $18-88$ years). According to the classification by the KDIGO criteria, 146 patients were identified as OLP with CKD, which translated to a prevalence of $14.3 \%$ (95\% CI, $12.3-16.6 \%$ ). Thus, the prevalence of CKD among the patients in our study was significantly $\left(\mathrm{P}<0.05 ; \chi^{2}\right.$ test) higher than that $(10.8 \%$; $95 \%$ CI, $10.2-11.3 \%)$ in the general Chinese population (11). Furthermore, the prevalence of eGFR $<60 \mathrm{~mL} / \mathrm{min} / 1.73 \mathrm{~m}^{2}$ and UACR $>30 \mathrm{mg} / \mathrm{g}$ in OLP patients were $2.5 \%$ (95\% CI, $1.7-3.7 \%$ ) and $13.0 \%$ (95\% CI, 11.1-15.2\%), respectively. However, the prevalence of eGFR $<60 \mathrm{~mL} / \mathrm{min} / 1.73 \mathrm{~m}^{2}$ and UACR $>30 \mathrm{mg} / \mathrm{g}$ in the general Chinese population were 
Table 1 Demographic, oral lesion score and renal function indicators of OLP patients without and with CKD

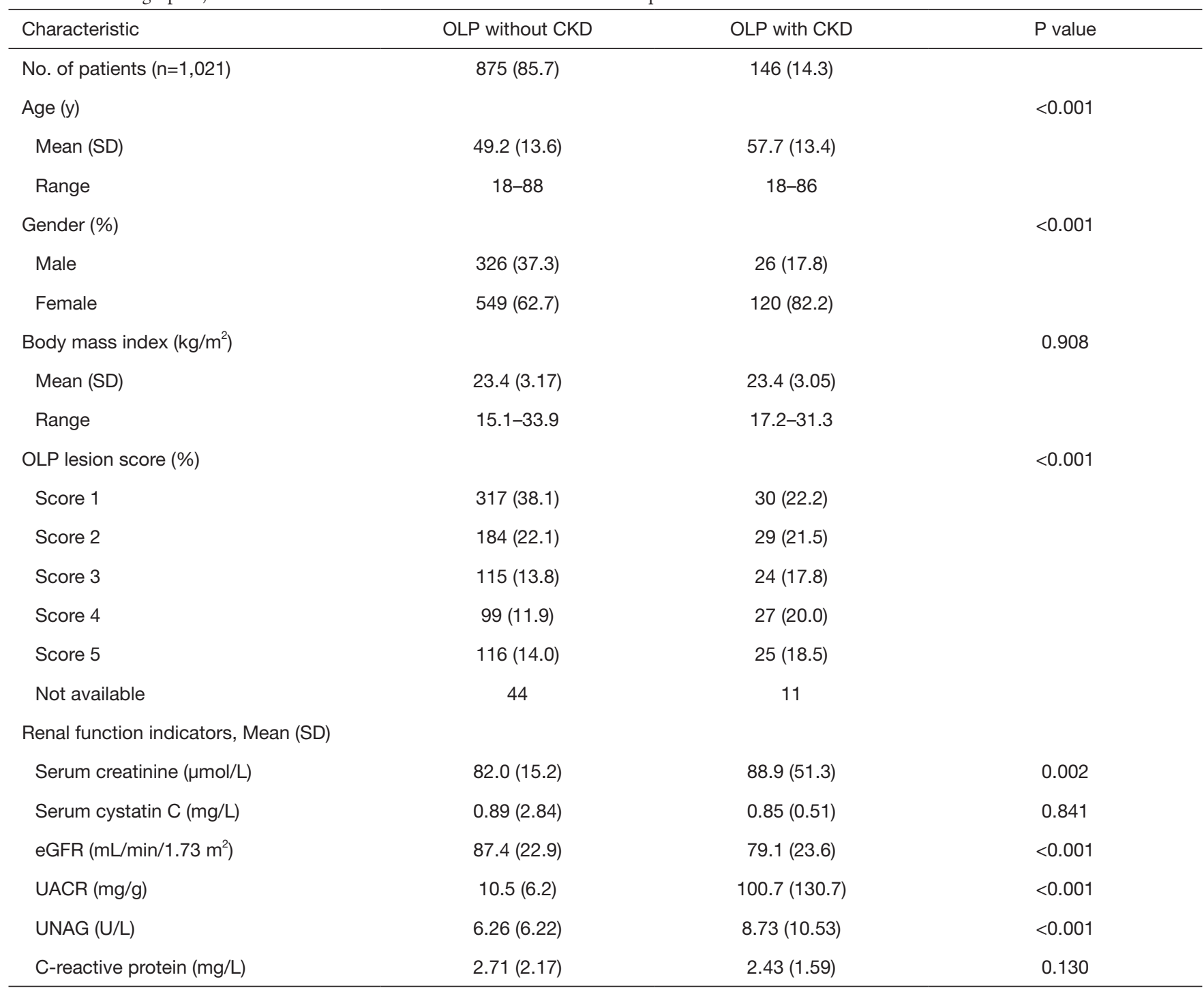

OLP, oral lichen planus; CKD, chronic kidney disease; SD, standard deviation; eGFR, estimated glomerular filtration rate; UACR, urine albumin-to-creatinine ratio; UNAG, Urine N-acetyl- $\beta$-D-glucosidase. Normal range values: serum creatinine, $62-115 \mu \mathrm{mol} / \mathrm{L}$; serum cystatin C, 0-1 mg/L; eGFR, 80-120 mL/min/1.73 m²; UACR, 0-30 mg/g; UNAG, 0.3-11.5 U/L; C-reactive protein, 0-10 mg/L.

$1.7 \%$ (95\% CI, $1.5-1.9 \%)$ and $9.4 \%(95 \%$ CI, $8.9-10.0 \%)$, respectively.

To determine the differences in demographics, oral lesion scores, and renal function indicators, a comparative analysis was performed between OLP patients without CKD ( $\mathrm{n}=875)$ and with CKD ( $\mathrm{n}=146)$ (Table 1). The mean age (57.7 years) of the patients with CKD was significantly higher than that (49.2 years) of the patients without CKD $(\mathrm{P}<0.001$; Student's $t$ test). The proportions of females and patients with OLP lesion scores 3-5 was higher in the CKD group than in the non-CKD group (both $\mathrm{P}<0.001, \chi^{2}$ test).
The mean values of serum creatinine, eGFR, UACR, and UNAG in patients with CKD were significantly higher than those in patients without CKD (all $\mathrm{P}<0.01$; Student's $t$ test).

\section{Renal function indicators and CKD staging correlated with the severity of $O L P$}

Oral lesion scores were available for 966 of the 1,021 patients with OLP in this study. To analyze the correlation between the severity of OLP and CKD, we compared renal function indicators and CKD staging in 3 groups: mild 
Table 2 Correlation of disease severity of OLP ( $n=966)$ with renal function indicators and CKD stage

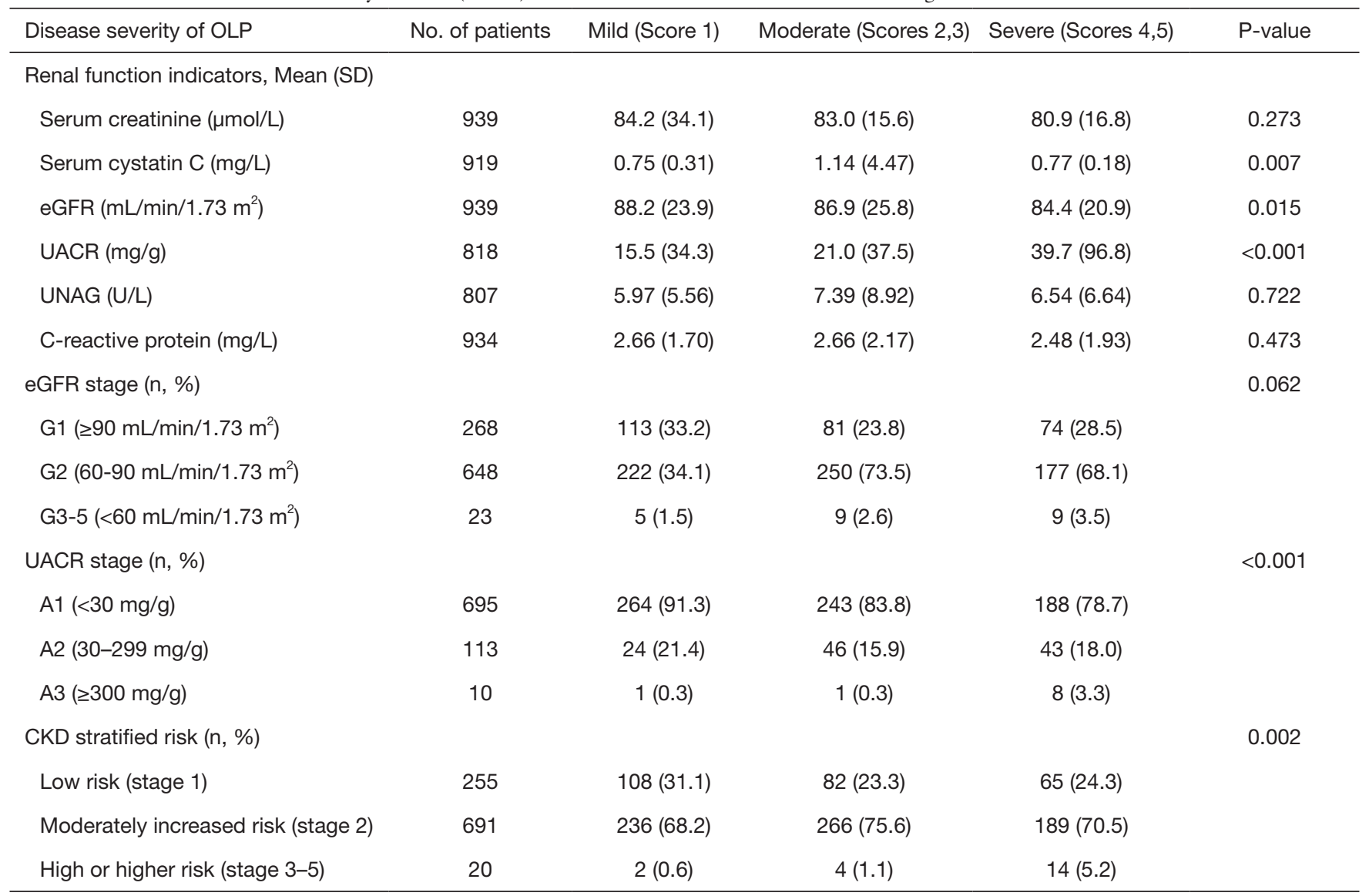

OLP, oral lichen planus; CKD, chronic kidney disease; SD, standard deviation; eGFR, estimated glomerular filtration rate; UACR, urine albumin-to-creatinine ratio; UNAG, Urine $\mathrm{N}$-acetyl- $\beta$-D-glucosidase.

(score: 1), moderate (scores: 2 and 3), and severe (score: 4 and 5) OLP (Table 2). The mean value of eGFR significantly decreased in order of mild, moderate, and severe OLP (88.2, 86.9 , and $84.4 \mathrm{~mL} / \mathrm{min} / 1.73 \mathrm{~m}^{2}$, respectively; $\mathrm{P}=0.015$ ). The mean value of UACR significantly increased in order of mild, moderate, and severe OLP $(15.5,21.0$, and $39.7 \mathrm{mg} / \mathrm{g}$, respectively; $\mathrm{P}<0.001$; Student's $t$ test). Consistently, UACR level was positively correlated with the severity of OLP $(\mathrm{P}<0.001$, Pearson's correlation), while eGFR level was marginally correlated with the severity of OLP $(\mathrm{P}=0.062$, Table 2). According to the KDIGO stratified risk criteria, CKD stage was also positively correlated with the severity of OLP $(\mathrm{P}<0.001$, Pearson's correlation).

\section{Risk assessment for CKD occurrence and relevant covariates in OLP}

Systemic comorbidity data on hypertension, diabetes, and
CVD were available for 675 of the 1,021 patients with OLP in this study. Among these 675 patients, 99 (14.7\%) were identified as OLP with CKD. There were no significant differences in demographics, BMI, or CKD prevalence or indicators between the 675 patients with systemic comorbidity data and the 346 patients without these data. Because the mean age of the patients with CKD was 57.7 years old, the patients were divided into two age groups ( $<58$ and $\geq 58$ years old) in the logistic analysis. We performed the statistical evaluation on the differences in $\mathrm{BMI}$ and the main lab parameters (serum creatinine, UACR, and eGFR) between the two age groups. The results revealed that $\mathrm{BMI}$ (mean, 23.4 vs. 23.5, $\mathrm{P}=0.435$ ) and serum creatinine (mean, 83.3 vs. $82.5, \mathrm{P}=0.239$ ) were not significantly difference between $<58$ - and $\geq 58$-year-old groups. Notably, eGFR (mean, 89.5 vs. 79.4, $\mathrm{P}=0.003$ ) and UACR (mean, 19.2 vs. 38.2, $\mathrm{P}<0.001$ ) were significantly difference between $<58$ - and $\geq 58$-year-old groups. These 
Table 3 Risk assessment for CKD occurrence and relevant covariates with emphasis on comorbidities in 675 patients with OLP

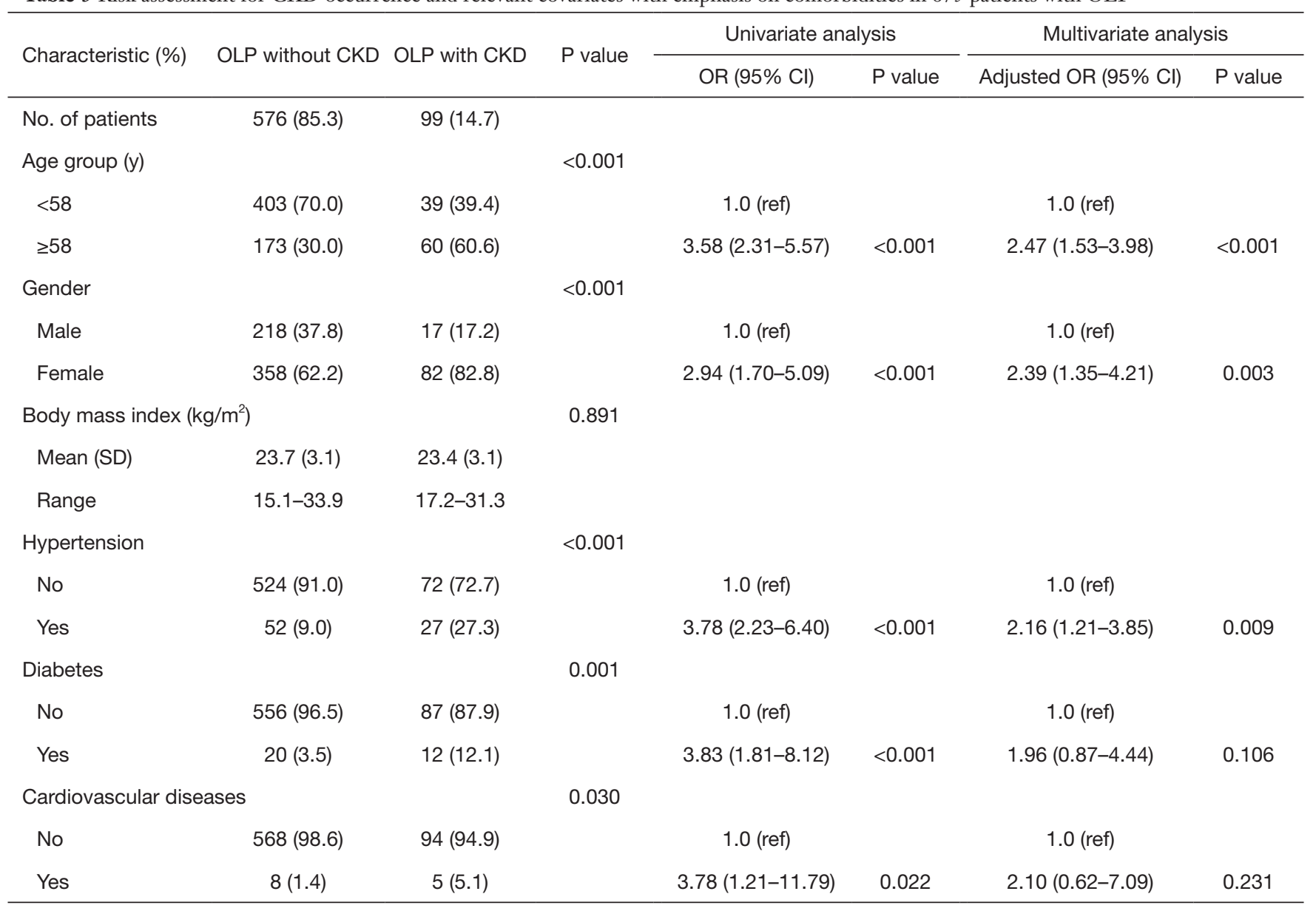

CKD, chronic kidney disease; OLP, oral lichen planus; SD, standard deviation; OR, odds ratio; Cl, confidence interval.

suggested that renal function should corelate with the age factor.

Univariate logistic analysis revealed that age ( $\geq 58$ years old), sex, hypertension, diabetes, and CVD were significant covariates (Table 3). Multivariate analysis revealed that the risk of CKD in OLP patients aged $\geq 58$ years old (adjusted OR, 2.47; 95\% CI, 1.53-3.98; $\mathrm{P}<0.001)$ was higher than that in patients aged $<58$ years old. Female OLP patients were at higher risk of CKD than were male patients (adjusted OR, 2.39; 95\% CI, 1.35-4.21; $\mathrm{P}=0.003)$. The risk of CKD was higher for patients with hypertension (adjusted OR, 2.16; 95\% CI, 1.21-3.8; $\mathrm{P}=0.009$ ) than for those without hypertension. However, diabetes and CVD were not found to be significant covariates, which indicated that neither of them is confounding variables of CKD occurrence in patients with OLP.

CKD is classified based on eGFR $<60 \mathrm{~mL} / \mathrm{min} / 1.73 \mathrm{~m}^{2}$ and UACR $>30 \mathrm{mg} / \mathrm{g}$. To further investigate whether the relevant covariates affect eGFR or UACR in patients with OLP, logistic regression models were also performed as per the method described above. Multivariate analysis revealed that only age ( $\geq 58$ years old; adjusted OR, 9.92; $95 \%$ CI, 2.08-47.23; $\mathrm{P}=0.004)$ was an independent risk factor for eGFR $<60 \mathrm{~mL} / \mathrm{min} / 1.73 \mathrm{~m}^{2}$. Furthermore, age ( $\geq 58$ years old; adjusted OR, 2.35 ; $95 \%$ CI, 1.42-3.90; $\mathrm{P}=0.001$ ), female sex (adjusted OR, 2.44; 95\% CI, 1.34-4.47; $\mathrm{P}=0.004$ ), and hypertension (adjusted OR, 2.31; 95\% CI, 1.27-4.21; $\mathrm{P}=0.006$ ) were independent risk factors for UACR $>30 \mathrm{mg} / \mathrm{g}$ in patients with OLP (Table 4). These results indicated that hypertension and female sex were independent factors of UACR $>30 \mathrm{mg} / \mathrm{g}$ and were further associated with the occurrence of CKD in OLP.

Based on the adjusted analyses, the adjusted prevalence of CKD in males $(7.2 \%)$, patients aged $<58$ years old $(8.8 \%)$, 
Table 4 Multivariate logistic analyses of risk factors of abnormal eGFR and UACR in OLP patients

\begin{tabular}{|c|c|c|c|c|}
\hline Characteristic & \multicolumn{2}{|c|}{ eGFR $<60 \mathrm{~mL} / \mathrm{min} / 1.73 \mathrm{~m}^{2}$} & \multicolumn{2}{|c|}{ UACR $>30 \mathrm{mg} / \mathrm{g}$} \\
\hline \multicolumn{5}{|l|}{ Age (y) } \\
\hline$<58$ & 1.0 (ref) & & 1.0 (ref) & \\
\hline$\geq 58$ & $9.92(2.08-47.23)$ & 0.004 & 2.35 (1.42-3.90) & 0.001 \\
\hline Male & 1.0 (ref) & & 1.0 (ref) & \\
\hline Female & $1.43(0.38-5.37)$ & 0.593 & $2.44(1.34-4.47)$ & 0.004 \\
\hline \multicolumn{5}{|l|}{ Hypertension } \\
\hline No & 1.0 (ref) & & 1.0 (ref) & \\
\hline No & 1.0 (ref) & & 1.0 (ref) & \\
\hline Yes & $2.60(0.63-10.79)$ & 0.187 & $2.03(0.87-4.73)$ & 0.100 \\
\hline \multicolumn{5}{|c|}{ Cardiovascular disease } \\
\hline No & 1.0 (ref) & & 1.0 (ref) & \\
\hline Yes & $0.00(0.00-0.00)$ & 0.999 & $2.22(0.63-7.82)$ & 0.217 \\
\hline
\end{tabular}

eGFR, estimated glomerular filtration rate; UACR, urine albumin-to-creatinine ratio; OLP, oral lichen planus; OR, odds ratio; Cl, confidence interval.

and patients without hypertension (10.8\%) were lower than the overall prevalence of CKD among patients with OLP (14.7\%; 95\% CI, 12.2-17.5\%). Conversely, the adjusted prevalence of CKD in females $(18.6 \%)$, patients aged $\geq 58$ years old $(25.8 \%)$, and patients with hypertension (34.2\%) were higher than the overall prevalence of CKD among patients with OLP (14.7\%; 95\% CI, 12.2-17.5\%).

\section{Discussion}

OLP is a chronic, relapsing disease often associated with systemic comorbidities. Comorbidities of OLP have been preliminarily elucidated and described, mainly in the fields of diabetes and CVD $(8,9)$. Recently, psoriasis has also been robustly demonstrated to be associated with CKD by some well-designed studies (15). Because OLP is an immunemediated inflammatory mucocutaneous disease $(1,2)$, it is reasonable to speculate that it could also be linked to CKD. However, few data are available regarding renal impairment in patients with OLP. The current study attempted to examine renal impairment using blood and urine tests and to elucidate the possible association between incident $\mathrm{CKD}$ and OLP in a large prospective series of patients from eastern China. We also assessed the risk factors for incident CKD in patients with OLP and the relevant covariates after adjustment for comorbidities including diabetes, hypertension, and CVD.

To the best of our knowledge, this study is the first to investigate the renal function indicators and the prevalence and staging of incident CKD in patients with OLP, as well as the disease severity. We performed a comparative analysis with the data from a nationally representative crosssectional survey of Chinese adults as a control group (11). Arguably, the mean ages of the OLP patients (50.4 years old) and the general Chinese adult group (49.6 years old) were comparable, and female predominance was found in both groups (11). According to the classification of the KDIGO criteria (28), the prevalence and demographic- and comorbidity-adjusted prevalence of CKD in patients with OLP were significantly higher than those in the general Chinese population (11). Importantly, we observed a significant positive correlation between the severity of OLP and renal function indicators and CKD stage (Table 2). Since the clinical manifestations and scoring of lesions in the oral 
mucosa can be observed relatively easily, it is plausible that the severity of OLP may be useful in detecting individuals at risk and the development of kidney damage, as well as in the staging of CKD. This also suggests that CKD prevention and interference could effectively reduce the incidence and/or severity of OLP.

OLP is frequently linked to systemic comorbidities, including hypertension, diabetes, and CVD $(8,9)$. In our study, $11.7 \%$ of patients had hypertension and $4.7 \%$ had diabetes; these rates were within the range of those reported for hypertension (11-27\%) (28-30) and diabetes (1.6-37.7\%) previously (8). Furthermore, the prevalence of patients with CVDs $(1.9 \%)$ in our study was lower than the prevalence of $7.8 \%$ reported in an Italian study (9). These comorbidities can also be observed in patients with CKD (10). The prevalence of comorbidities and risk factors for CKD in OLP can be confounding. Hence, a multivariate logistic regression model, adjusted for age at diagnosis, sex, hypertension, diabetes, and CVD, was performed. Multivariate regression analysis revealed that age $\geq 58$ years old, female sex, and hypertension were independent risk factors for incident CKD in patients with OLP, while diabetes and CVD were not significant factors. Furthermore, multivariate logistic analyses of risk factors for abnormal eGFR and UACR, as the 2 determinants of CKD occurrence, were also performed. We found that female sex and hypertension were independent risk factors for normal eGFR $\left(<60 \mathrm{~mL} / \mathrm{min} / 1.73 \mathrm{~m}^{2}\right)$ but abnormal UACR $(>30 \mathrm{mg} / \mathrm{g}$ ), while age $\geq 58$ years old was an independent risk factor for both. Taken together, these results suggest that hypertension prevention and interference may effectively decrease the incidence of CKD in patients with OLP.

We acknowledge that there are limitations to this study. First, this was a cross-sectional study, which makes inference of a causal relationship between indicators of kidney damage and associated factors impossible. Second, data on systemic comorbidities were not available for all participants, although there were no significant differences in baseline factors or renal function indicators between the patients with and without available data. Third, the calculation of the estimated sample size needed for the study was based on the prevalence of OLP in the general population $(3,31)$, and there was a lack of a contemporaneous control group with which to compare the prevalence of CKD. We adopted national cross-sectional data from Chinese adults with a plausible age- and sex-matched population as the control group (11). Our study is the first to investigate the occurrence and staging of CKD in patients with
OLP. However, the patients included only the Chinese population, which limits the generalizability of the results to other ethnicities and races. Our results therefore need to be confirmed by large-scale studies conducted in different geographic and ethnic populations.

In conclusion, this study identified for the first time that CKD is a new incident comorbidity in patients with OLP, with incident CKD occurrence and staging exhibiting significant associations with OLP and its severity. Further studies are necessary to determine whether CKD prevention and interference can effectively reduce the incidence and/or severity of OLP. Additionally, further research is required into the mechanisms underlying the coexistence of OLP and CKD.

\section{Acknowledgments}

The authors thank AME Editing Service for language editing.

Funding: This work was funded by National Natural Science Foundation of China (82020108010, 81970937), the Chinese Stomatological Association (CSA-Z2015-03), and Shanghai Municipal Health Committee (20174Y0098).

\section{Footnote}

Reporting Checklist: The authors have completed the STROBE reporting checklist. Available at http://dx.doi. org/10.21037/atm-21-699

Data Sharing Statement: Available at http://dx.doi. org/10.21037/atm-21-699

Conflicts of Interest: All authors have completed the ICMJE uniform disclosure form (available at http://dx.doi. org/10.21037/atm-21-699). The authors have no conflicts of interest to declare.

Ethical Statement: The authors are accountable for all aspects of the work in ensuring that questions related to the accuracy or integrity of any part of the work are appropriately investigated and resolved. This study was approved by the Institutional Review Board of Shanghai Ninth People's Hospital, Shanghai Jiao Tong University School of Medicine (No. (2016)01). Written informed consent was obtained from all study participants. The study was conducted in accordance with the Declaration of Helsinki (as revised in 2013). 
Open Access Statement: This is an Open Access article distributed in accordance with the Creative Commons Attribution-NonCommercial-NoDerivs 4.0 International License (CC BY-NC-ND 4.0), which permits the noncommercial replication and distribution of the article with the strict proviso that no changes or edits are made and the original work is properly cited (including links to both the formal publication through the relevant DOI and the license). See: https://creativecommons.org/licenses/by-nc-nd/4.0/.

\section{References}

1. DeAngelis LM, Cirillo N, McCullough MJ. The immunopathogenesis of oral lichen planus-Is there a role for mucosal associated invariant T cells? J Oral Pathol Med 2019;48:552-9.

2. Yang JY, Tan YQ, Zhou G. T cell-derived exosomes containing cytokines induced keratinocytes apoptosis in oral lichen planus. Oral Dis 2021. [Epub ahead of print]. doi: 10.1111/odi.13795.

3. McCartan BE, Healy CM. The reported prevalence of oral lichen planus: a review and critique. J Oral Pathol Med 2008;37:447-53.

4. Zucoloto ML, Shibakura MEW, Pavanin JV, et al. Severity of oral lichen planus and oral lichenoid lesions is associated with anxiety. Clin Oral Investig 2019; 23:4441-8.

5. Lodi G, Carrozzo M, Furness S, et al. Interventions for treating oral lichen planus: a systematic review. Br J Dermatol 2012;166:938-47.

6. González-Moles MÁ, Ruiz-Ávila I, González-Ruiz L, et al. Malignant transformation risk of oral lichen planus: A systematic review and comprehensive meta-analysis. Oral Oncol 2019;96:121-30.

7. Giuliani M, Troiano G, Cordaro M, et al. Rate of malignant transformation of oral lichen planus: A systematic review. Oral Dis 2019;25:693-709.

8. Otero Rey EM, Yáñez-Busto A, Rosa Henriques IF, et al. Lichen planus and diabetes mellitus: Systematic review and meta-analysis. Oral Dis 2019;25:1253-64.

9. Conrotto D, Barattero R, Carbone M, et al. Can atrophicerosive oral lichen planus promote cardiovascular diseases? A population-based study. Oral Dis 2018;24:215-8.

10. Gajjala PR, Sanati M, Jankowski J. Cellular and molecular mechanisms of chronic kidney disease with diabetes mellitus and cardiovascular diseases as its comorbidities. Front Immunol 2015;6:340.

11. Zhang L, Wang F, Wang L, et al. Prevalence of chronic kidney disease in China: a cross-sectional survey. Lancet
2012;379:815-22.

12. Deschamps-Lenhardt S, Martin-Cabezas R, Hannedouche $\mathrm{T}$, et al. Association between periodontitis and chronic kidney disease: Systematic review and meta-analysis. Oral Dis 2019;25:385-402.

13. Zhao D, Khawaja AT, Jin L, et al. Effect of non-surgical periodontal therapy on renal function in chronic kidney disease patients with periodontitis: a systematic review and meta-analysis of interventional studies. Clin Oral Investig 2020;24:1607-18.

14. Raksasuk S, Ungprasert P. Patients with rheumatoid arthritis have an increased risk of incident chronic kidney disease: a systematic review and meta-analysis of cohort studies. Int Urol Nephrol 2020;52:147-54.

15. Ungprasert $P$, Raksasuk S. Psoriasis and risk of incident chronic kidney disease and end-stage renal disease: a systematic review and meta-analysis. Int Urol Nephrol 2018;50:1277-83.

16. Shahbazi M, Smailnejad Ganji K, Mirzakhani M, et al. The role of immune response in initiation and progression of chronic kidney disease. Iran J Kidney Dis 2019;13:283-99.

17. Espi M, Koppe L, Fouque D, et al. Chronic Kidney Disease-Associated Immune Dysfunctions: Impact of Protein-Bound Uremic Retention Solutes on Immune Cells. Toxins (Basel) 2020;12:300.

18. Winterberg PD, Ford ML. The effect of chronic kidney disease on T cell alloimmunity. Curr Opin Organ Transplant 2017;22:22-8.

19. Lioulios G, Fylaktou A, Papagianni A, et al. T cell markers recount the course of immunosenescence in healthy individuals and chronic kidney disease. Clin Immunol 2021;225:108685.

20. Oguz U, Takci Z, Oguz ID, et al. Are patients with lichen planus really prone to urolithiasis? Lichen planus and urolithiasis. Int Braz J Urol 2016;42:571-7.

21. Halevy S, Arie R, Ingber A, et al. Analysis of lithogenous factors in lichen planus. Acta Derm Venereol 1990;70:441-2.

22. van der Meij EH, van der Waal I. Lack of clinicopathologic correlation in the diagnosis of oral lichen planus based on the presently available diagnostic criteria and suggestions for modifications J Oral Pathol Med 2003;32:507-12.

23. Sarnak MJ, Bloom R, Muntner P, et al. KDOQI US commentary on the 2013 KDIGO Clinical Practice Guideline for Lipid Management in CKD. Am J Kidney Dis 2015;65:354-66.

24. Thongprasom K, Luangjarmekorn L, Sererat T, et al. Relative efficacy of fluocinolone acetonide compared with 


\section{Page 10 of 10}

triamcinolone acetonide in treatment of oral lichen planus. J Oral Pathol Med 1992;21:456-8.

25. Lopez Jornet P, Aznar-Cayuela C. Efficacy of topical chamomile management vs. placebo in patients with oral lichen planus: a randomized double-blind study. J Eur Acad Dermatol Venereol 2016;30:1783-6.

26. Lajevardi V, Ghodsi SZ, Hallaji Z, et al. Treatment of erosive oral lichen planus with methotrexate. J Dtsch Dermatol Ges 2016;14:286-93.

27. Lavaee F, Shadmanpour M. Comparison of the effect of photodynamic therapy and topical corticosteroid on oral lichen planus lesions. Oral Dis 2019;25:1954-63.

28. Cassol-Spanemberg J, Blanco-Carrión A, Rodríguez-de Rivera-Campillo ME, et al. Cutaneous, genital and oral lichen planus: A descriptive study of 274 patients. Med

Cite this article as: Deng Y, Wang C, Shen Y, Shen B, Ding F, Tang G, Liu W. Prevalence and risk of chronic kidney disease in oral lichen planus: a large cross-sectional study from eastern China. Ann Transl Med 2021;9(13):1078. doi: 10.21037/atm21-699

\section{Deng et al. Chronic kidney disease and oral lichen planus}

Oral Patol Oral Cir Bucal 2019;24:e1-7.

29. Munde AD, Karle RR, Wankhede PK, et al. Demographic and clinical profile of oral lichen planus: A retrospective study. Contemp Clin Dent 2013;4:181-5.

30. Barbosa NG, Silveira ÉJ, Lima EN, et al. Factors associated with clinical characteristics and symptoms in a case series of oral lichen planus. Int J Dermatol 2015;54:e1-6.

31. Li C, Tang X, Zheng X, et al. Global prevalence and incidence estimates of oral lichen planus: a systematic review and meta-analysis. JAMA Dermatol 2020;156:172-81.

(English Language Editors: C. Betlazar-Maseh and J. Reynolds) 

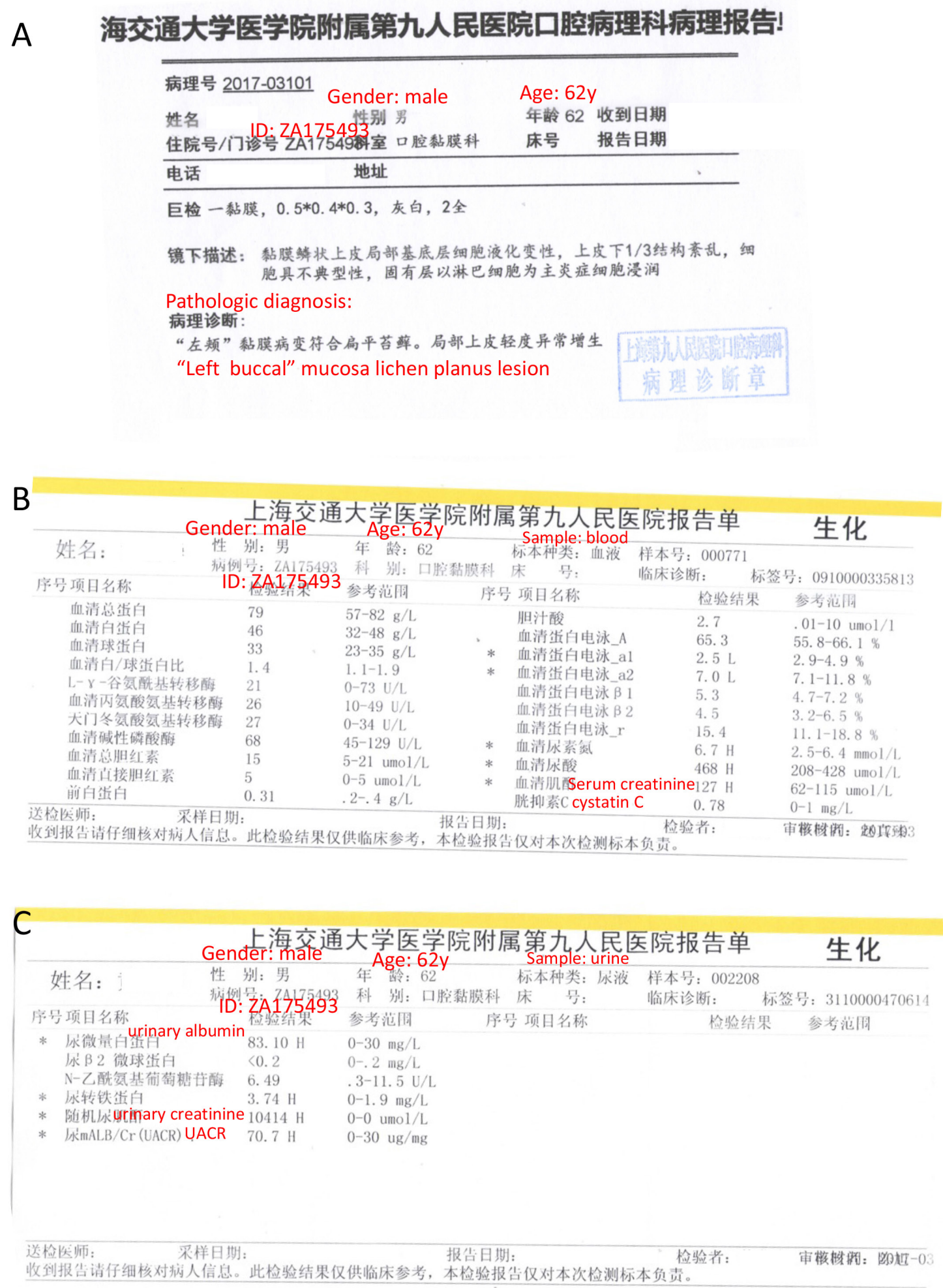

Figure S1 A representative male case of oral buccal lichen planus concomitant chronic kidney disease. (A) Pathological report shows the diagnosis of oral lichen planus. (B) Renal function report tested in blood sample shows abnormal serum creatinine (127 $\mu$ mol/L). (C) Renal function report tested in urine sample shows abnormal UACR $(70.7 \mu \mathrm{g} / \mathrm{mg})$. 


\section{A \\ 海交通大学医学院附属第九人民医院口腔病理科病理报告!}

\begin{tabular}{|c|c|}
\hline 病理号 2018-00944 Gender: female & Age: 70y \\
\hline 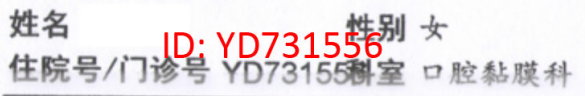 & $\begin{array}{ll}\text { 年龄 } 70 & \text { 收到日期 } \\
\text { 床号 } \quad \text { 报告日期 }\end{array}$ \\
\hline
\end{tabular}

巨检一带秥膜组织, $0.5 \times 0.4 \times 0.3$, 灰黄

镜下描述: 基底层细胞液化变性, 固有层淋巴细胞浸润

Pathologic diagnosis:

病理诊断:

“左舌缘”粘膜扁平苔䱣。

"Left tongue" mucosa lichen planus

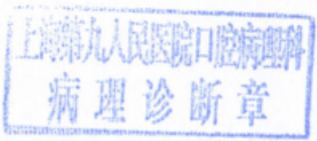

B

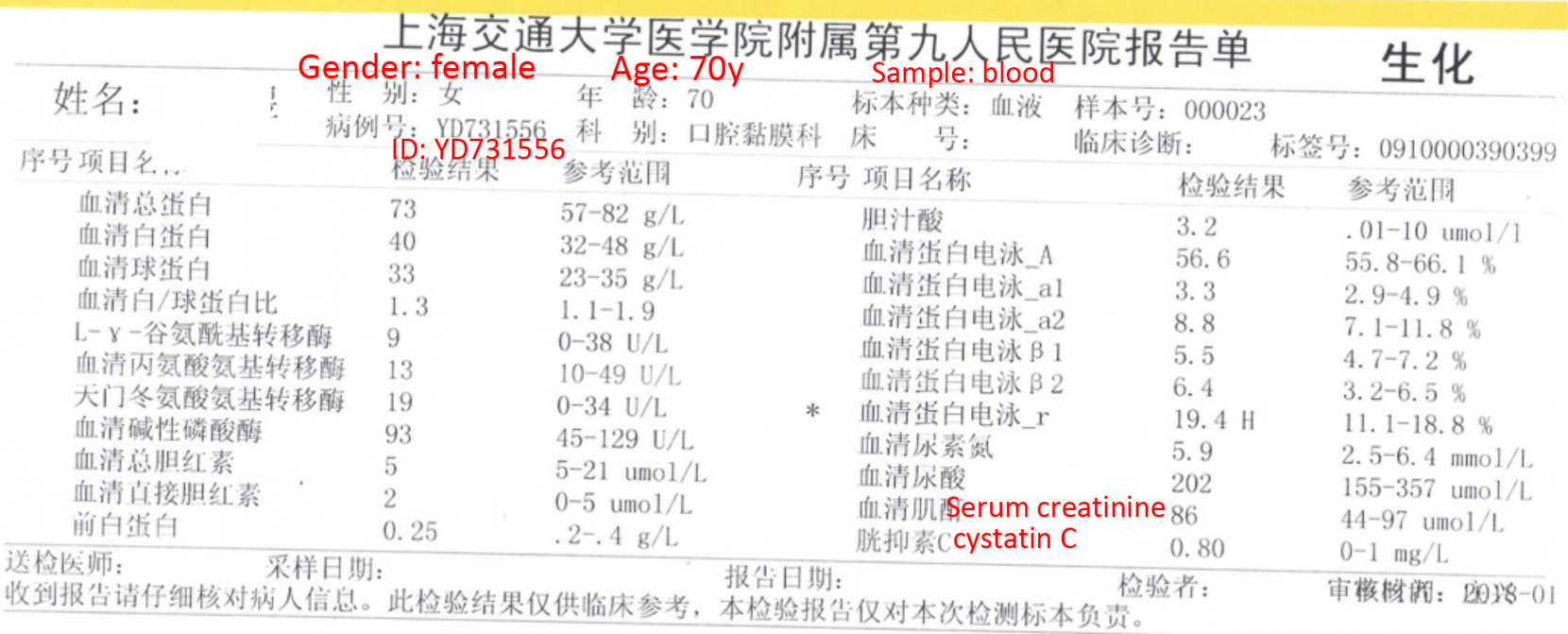

\section{C}

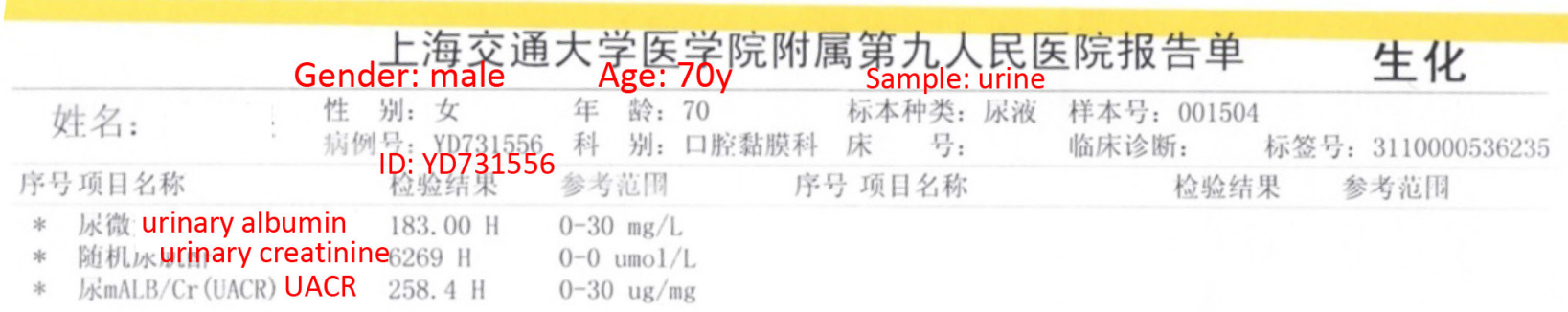

Figure S2 A representative female case of oral tongue lichen planus concomitant chronic kidney disease. (A) Pathological report shows the diagnosis of oral lichen planus. (B) Renal function report tested in blood sample shows normal serum creatinine (86 $\mu$ mol/L). (C) Renal function report tested in urine sample shows abnormal UACR $(258.4 \mu \mathrm{g} / \mathrm{mg})$. 